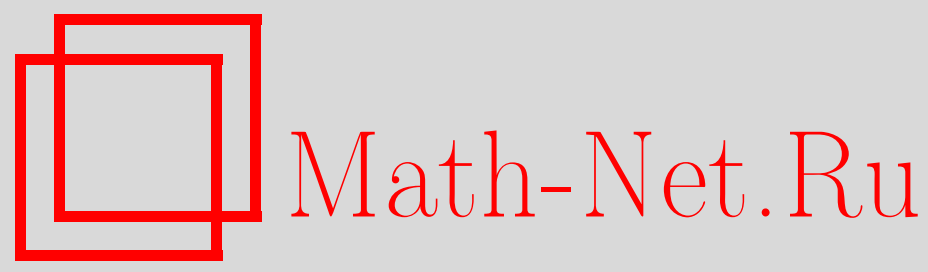

Б. В. Пальцев, О равномерных по действительным аргументу и индексу двусторонних оценках для модифицированных функций Бесселя, Матем. заметки, 1999, том 65, выпуск 5, 681-692

DOI: https://doi.org/10.4213/mzm1100

Использование Общероссийского математического портала Math-Net.Ru подразумевает, что вы прочитали и согласны с пользовательским соглашением http://www.mathnet.ru/rus/agreement

Параметры загрузки:

IP : 18.234 .156 .22

26 апреля 2023 г., 12:28:08

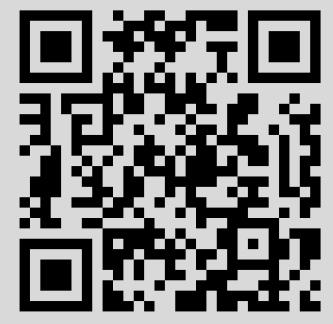




\section{О РАВНОМЕРНЫХ ПО ДЕЙСТВИТЕЛЬНЫМ АРГУМЕНТУ И ИНДЕКСУ ДВУСТОРОННИХ ОЦЕНКАХ ДЛЯ МОДИФИЦИРОВАННЫХ ФУНКЦИЙ БЕССЕЛЯ}

\section{Б. В. Пальцев}

Устанавливаются равномерные по действительным аргументу и индексу двусторонние оценки для функций $a_{\nu}(x)=x I_{\nu}^{\prime}(x) / I_{\nu}(x)$ и $b_{\nu}(x)=x K_{\nu}^{\prime}(x) / K_{\nu}(x)$, а также для самих модифицированных функций Бесселя $I_{\nu}(x)$ и $K_{\nu}(x)$ в четверти $x>0, \nu \geqslant 0$ за исключением некоторых окрестностей точки $x=0, \nu=0$.

Библиография: 10 названий.

Введение. Настоящая работа возникла в процессе обоснования быстросходящихся итерационных методов с расщеплением граничных условий решения первой краевой задачи для сингулярно возмущенной системы типа Стокса в областях, обладающих круговой симметрией (круг, кольцо, шар, шаровой слой). Такие методы были предложены в [1]-[3] и исследованы там для случая области, представляющей собой слой между двумя гиперплоскостями, при условии периодичности по ортогональным направлениям, параллельным граничным гиперплоскостям. В [4] аналогичньй итерационньй метод с расщеплением граничных условий для сингулярного уравнения типа бигармонического был предложен и исследован для случая шара в $\mathbb{R}^{n}$.

Для обоснования этих методов в случае области, представляющей собой кольцо или шаровой слой, необходимы равномерные оценки по действительным аргументу $x$ и индексу $\nu$ функций

$$
a_{\nu}(x)=\frac{x I_{\nu}^{\prime}(x)}{I_{\nu}(x)} \quad \text { и } b_{\nu}(x)=\frac{x K_{\nu}^{\prime}(x)}{K_{\nu}(x)}
$$

на множествах $D_{r_{*}}=\left\{(x, \nu): x>0, \nu \geqslant 0, \sqrt{x^{2}+\nu^{2}} \geqslant r_{*}\right\}, r_{*}>0$, где $I_{\nu}(x)$ и $K_{\nu}(x)$ - модифицированные функции Бесселя первого и третьего рода соответственно (последние - функции Макдональда), а также равномерные оценки на тех же множествах и самих функций $I_{\nu}(x)$ и $K_{\nu}(x)$. Последние образуют фундаментальную систему решений дифференциального уравнения

$$
x^{2} u^{\prime \prime}+x u^{\prime}-\left(x^{2}+\nu^{2}\right) u=0, \quad x>0,
$$

Работа выполнена при поддержке Российского фонда фундаментальных исследований, гранты № 96-01 -00732а, № 99-01-00852. 
причем $I_{\nu}(x)$ - ограниченное в нуле решение уравнения (2), имеющее асимптотическое поведение

$$
I_{\nu}(x) \sim \frac{e^{x}}{\sqrt{2 \pi x}}\left(1+O\left(x^{-1}\right)\right), \quad x \rightarrow \infty,
$$

а $K_{\nu}(x)$ - экспоненциально убьвающее с ростом $x$ решение $(2)$, имеющее асимптотическое поведение

$$
K_{\nu}(x) \sim \sqrt{\frac{\pi}{2 x}} e^{-x}\left(1+O\left(x^{-1}\right)\right), \quad x \rightarrow \infty
$$

(см., например, [5], [6]).

Исследованию асимптотического поведения функций Бесселя, в частности и модифицированных, посвящена обширная литература. Для функций $I_{\nu}(x)$ и $K_{\nu}(x)$, а также их производных хорошо известны асимптотические разложения при больших значениях аргумента и ограниченных значениях индекса, равномерные асимптотические разложения при больших значениях индекса, а также некоторые другие разложения. Однако необходимые для указанных вьше целей равномерные по $x$ и $\nu$ двусторонние оценки для функций $I_{\nu}(x)$ и $K_{\nu}(x)$, а также для функций $(1)$ в известных руководствах и работах по бесселевым функциям (см, например, [5]-[9]) обнаружить на удалось.

Между тем, нужные оценки, о которых идет речь, для функций (1) могут быть получены с помощью простой техники, основанной на использовании теоремы о дифференциальном неравенстве (см., например, [10]) и построении уточненных верхних и нижних барьеров (при сушественном использовании лишь для фиксированных значений $\nu$ степенного разложения в нуле до $O\left(x^{6}\right)$ функции $a_{\nu}(x)$ и асимптотического разложения при $x \rightarrow \infty$ до $O\left(x^{-3}\right)$ функции $\left.b_{\nu}(x)\right)$. Отметим, что такой подход был использован в [4] для получения грубой оценки сверху для функции $a_{\nu}(x)$. Необходимыеже двусторонние равномерные оценки для функций $I_{\nu}(x)$ и $K_{\nu}(x)$ получаются уже простьп интегрированием уточненных оценок для функций (1) с учетом нормировок (3) и (4). В настоящей статье проводится реализация этого плана и устанавливаются необходимые для указанных вьше целей оценки функций $(1), I_{\nu}(x)$ и $K_{\nu}(x)$.

1. Основные оценки. Сформулируем здесь устанавливаемые ниже в п. 3 основные оценки для функций (1), а также устанавливаемые в п. 4 основные оценки для функций $I_{\nu}(x)$ и $K_{\nu}(x)$.

ТЕорема 1. Для функиий (1) имеют место следующие двусторонние оценки. Для $a_{\nu}(x)$ :

$$
-\frac{x^{2}}{2\left(x^{2}+\nu^{2}\right)^{3 / 2}}<a_{\nu}(x)-\sqrt{x^{2}+\nu^{2}}+\frac{x^{2}}{2\left(x^{2}+\nu^{2}\right)}<\frac{x^{2}}{2\left(x^{2}+\nu^{2}\right)^{3 / 2}},
$$

причем оценка сверху выполняется для любых $x>0, \nu \geqslant 0$, а оценка снизу - на мнохсестве

$$
\left\{(x, \nu): x>0, \nu \geqslant \frac{1}{2}\right\} \cup\left\{(x, \nu): x>0, \nu \geqslant 0, \sqrt{x^{2}+\nu^{2}} \geqslant \frac{(\sqrt{7}+2)}{3}\right\} .
$$

Для $b_{\nu}(x)$ :

$$
-\frac{\beta x^{2}}{\left(x^{2}+\nu^{2}\right)^{3 / 2}}<b_{\nu}(x)+\sqrt{x^{2}+\nu^{2}}+\frac{x^{2}}{2\left(x^{2}+\nu^{2}\right)}<\frac{x^{2}}{8\left(x^{2}+\nu^{2}\right)^{3 / 2}},
$$


$\beta>1 / 2$ - произвольное, причем оценка сверху выполняется на множестве

$$
\left\{(x, \nu): x>0, \nu \geqslant 0, \sqrt{x^{2}+\nu^{2}} \geqslant \frac{1}{5}\right\}
$$

а оценка снизу - на множестве

$$
\left\{(x, \nu): x>0, \nu \geqslant 0, \sqrt{x^{2}+\nu^{2}} \geqslant \frac{2 \beta}{(2 \beta-1)}\right\} .
$$

ТЕОРема 2. Для модифицированных функиий Бесселя имеют место следующие двусторонние оченки. Для $I_{\nu}(x)$ :

$$
e^{-\frac{1}{2 \sqrt{x^{2}+\nu^{2}}}} \leqslant I_{\nu}(x) \sqrt{2 \pi}\left(x^{2}+\nu^{2}\right)^{1 / 4} e^{-\left(\sqrt{x^{2}+\nu^{2}}+\nu \ln \frac{x}{\nu+\sqrt{x^{2}+\nu^{2}}}\right)} \leqslant e^{\frac{1}{2 \sqrt{x^{2}+\nu^{2}}}},
$$

причем оценка снизу выполняется для любы $x>0, \nu \geqslant 0$, а оценка сверху - на мнохестве (6). Для $K_{\nu}(x)$ :

$$
e^{-\frac{1}{8 \sqrt{x^{2}+\nu^{2}}}} \leqslant K_{\nu}(x) \sqrt{\frac{\pi}{2}}\left(x^{2}+\nu^{2}\right)^{1 / 4} e^{\left(\sqrt{x^{2}+\nu^{2}}+\nu \ln \frac{x}{\nu+\sqrt{x^{2}+\nu^{2}}}\right)} \leqslant e^{\frac{\beta}{\sqrt{x^{2}+\nu^{2}}}}
$$

$\beta>1 / 2$ - произвольное, причем нижняя оченка выполняется на мнохестве (8), а верхняя - на множестве (9).

2. Простейшие двусторонние оценки для функций (1). Каждая из функций $a_{\nu}(x)$ и $b_{\nu}(x)$ удовлетворяют следующему уравнению Риккати:

$$
v_{\nu}^{\prime}(x)=-\frac{1}{x}\left(v_{\nu}(x)\right)^{2}+\frac{x^{2}+\nu^{2}}{x} \stackrel{\text { def }}{=} F_{\nu}\left(v_{\nu}(x), x\right), \quad x>0 .
$$

Для получения двусторонних оценок для функций (1) воспользуемся следующим достаточным для нас вариантом известной из теории обыкновенных дифференциальных уравнений теоремы о дифференциальном неравенстве (см., например, [10, гл. III, § 4]).

ТЕОрема (о дифференциальном неравенстве). Пусть $F(v, x)$ - непрерывная функиия переменных $v$ и в в некоторой области $G$.

$\left.1^{0}\right)$ (В сторону возрастания $x$ ). Пусть $v(x)$ - решение на отрезке $\left[x_{0}, x_{0}+\delta\right]$, $\delta>0$, задачи Коши

$$
v^{\prime}(x)=F(v(x), x), \quad v\left(x_{0}\right)=v_{0}, \quad\left(v_{0}, x_{0}\right) \in G
$$

и пусть существует дифферениируемая на $\left[x_{0}, x_{0}+\delta\right]$ функиия $v_{+}(x)\left(u л и v_{-}(x)\right)$, удовлетворяющая неравенствам

$$
\begin{aligned}
& v_{+}^{\prime}(x)>F\left(v_{+}(x), x\right) \quad\left(\text { или } \quad v_{-}^{\prime}(x)<F\left(v_{-}(x), x\right)\right) \quad \forall x \in\left(x_{0}, x_{0}+\delta\right], \\
& v_{+}\left(x_{0}\right)>v\left(x_{0}\right) \quad\left(\text { uлu } \quad v_{-}\left(x_{0}\right)<v\left(x_{0}\right)\right) .
\end{aligned}
$$

Тогда $\forall x \in\left[x_{0}, x_{0}+\delta\right]$

$$
v(x)<v_{+}(x) \quad\left(\text { или соответственно } \quad v_{-}(x)<v(x)\right) .
$$


$\left.2^{0}\right)$ (В сторону убывания $\left.x\right)$. Пусть $v(x)$ - решение на отрезке $\left[x_{0}-\delta, x_{0}\right], \delta>0$, задачи Коши (13) и пусть существует дифферениируемая на $\left[x_{0}-\delta, x_{0}\right]$ функиия $v_{+}(x)\left(\right.$ или $\left.v_{-}(x)\right)$, удовлетворяющая неравенствам

$$
\begin{aligned}
& v_{+}^{\prime}(x)<F\left(v_{+}(x), x\right) \quad\left(\text { uли } \quad v_{-}^{\prime}(x)>F\left(v_{-}(x), x\right)\right) \quad \forall x \in\left[x_{0}-\delta, x_{0}\right],
\end{aligned}
$$

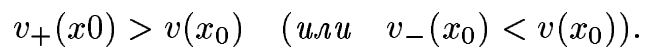

Тогда $\forall x \in\left[x_{0}-\delta, x_{0}\right]$ выполняется (15).

Функции $v_{+}(x)$ и $v_{-}(x)$, фигурирующие в этой теореме, будем назьвать соответственно верхним и нижним барьерами для решения $v(x)$.

Построение верхних и нижних барьеров для функций (1) как решений уравнения (12) удается осуществлять на основе применения: для $a_{\nu}(x)$ - части $1^{0}$ ) (в сторону возрастания $x$ ), а для $b_{\nu}(x)$ - части $2^{0}$ ) (в сторону убьвания $x$ ) вьшесформулированной теоремы.

Для таких построений потребуются следующие свойства функций (1):

$$
a_{\nu}(x)>0, \quad b_{\nu}(x)<0, \quad \forall x>0, \quad \nu \geqslant 0 .
$$

Положительность $a_{\nu}(x)$ следует, например, из представления

$$
a_{\nu}(x)=\nu+x \frac{I_{\nu+1}(x)}{I_{\nu}(x)}
$$

вытекающего из рекуррентного соотношения

$$
I_{\nu}^{\prime}(x)=I_{\nu+1}(x)+\frac{\nu}{x} I_{\nu}(x)
$$

(см. [6, формулы 9.6.26]) и положительности функций $I_{\nu}(x)$. Отрицательность $b_{\nu}(x)$ вытекает из непосредственного определения $(1)$, положительности функций $K_{\nu}(x)$ и отрицательности их производных $K_{\nu}^{(1)}(x)$ (что следует, например, из интегрального представления 9.6.24 в [6]).

Для $a_{\nu}(x)$ и $b_{\nu}(x)$ имеем

$$
a_{\nu}(x)=\nu+\frac{x^{2}}{2(\nu+1)}-\frac{x^{4}}{8(\nu+1)^{2}(\nu+2)}+O\left(x^{6}\right) \text { при } x \rightarrow 0,
$$

что получается, например, из (18) с использованием степенного представления для $I_{\nu}(x)$ (см. [6, формула 9.6.10]);

$$
b_{\nu}(x)=-x-\frac{1}{2}-\frac{4 \nu^{2}-1}{8} \cdot \frac{1}{x}+\frac{4 \nu^{2}-1}{8} \cdot \frac{1}{x^{2}}+O\left(x^{-3}\right) \text { при } x \rightarrow \infty .
$$

Это разложение получается, например, с использованием асимптотических разложений 9.7 .2 и 9.7 .4 в [6] для $K_{\nu}(x)$ и $K_{\nu}^{\prime}(x)$.

В этом пункте будут получены для начала грубые барьеры для функций (1). Полученные с их помощью простейшие оценки для этих функций тем не менее оказываются также полезнњми для указанных во введении целей. 
I. Верхний барьер для $a_{\nu}(x)$. Таким барьером для $a_{\nu}(x)$ на любом полуотрезке $\left[x_{0}, \infty\right)$, где $0<x_{0} \leqslant x_{*}(\nu), x_{*}(\nu)$ - достаточно малое (зависящее от $\nu \geqslant 0$ ), будет функция $v_{\nu,+}(x)=\sqrt{x^{2}+\nu^{2}}$.

Действительно, $v_{\nu,+}^{\prime}(x)=x / \sqrt{x^{2}+\nu^{2}}$, в то время как $F_{\nu}\left(v_{\nu,+}(x), x\right) \equiv 0$, а потому $v_{\nu,+}^{\prime}(x)>F_{\nu}\left(v_{\nu,+}(x), x\right) \forall x>0$. Кроме того, сравнивая разложения (19) для $a_{\nu}(x)$ и $v_{\nu,+}(x)=\nu+x^{2} /(2 \nu)+O\left(x^{4}\right)$ при $x \rightarrow \infty, \nu \neq 0$, а также выражение $v_{0,+}(x)=x$, нетрудно убедиться, что при достаточно малых $x_{0}>0$ вьполняется также и неравенство $v_{\nu,+}\left(x_{0}\right)>a_{\nu}\left(x_{0}\right)$. Таким образом, применяя часть $\left.1^{0}\right)$ теоремы о дифференциальном неравенстве, заключаем, что $a_{\nu}(x)<\sqrt{x^{2}+\nu^{2}}$.

II. Построение нижнего барьера для $a_{\nu}(x)$. Этот барьер будем искать опять же на полуинтервале $\left[x_{0}, \infty\right)$, где $x_{0}>0$, вообще говоря, зависит от $\nu$, в виде $v_{\nu,-}(x)=$ $\sqrt{x^{2}+\nu^{2}}-\alpha, \alpha>0$.

Число $\alpha$ нужно подобрать в первую очередь таким, чтобы неравенство $v_{\nu,-}^{\prime}(x)<$ $F_{\nu}\left(v_{\nu,-}(x), x\right)$ вьполнялось $\forall x>x_{0}$. Это неравенство, как легко видеть, эквивалентно неравенству

$$
(2 \alpha-1)\left(x^{2}+\nu^{2}\right)-\alpha^{2} \sqrt{x^{2}+\nu^{2}}+\nu^{2}>0 .
$$

Оказывается удачным положить $\alpha=1$. Тогда последнее неравенство принимает вид $\left(x^{2}+\nu^{2}\right)-\sqrt{x^{2}+\nu^{2}}+\nu^{2}>0$, и оно заведомо вьполняется при $\sqrt{x^{2}+\nu^{2}}>1$. Поэтому $x_{0}>0$ возьмем при $0 \leqslant \nu<1$ таким, что $\sqrt{x_{0}^{2}+\nu^{2}}=1$, а при $\nu \geqslant 1-$ лишш достаточно малым. При таких $x_{0}$ будет вьполняться и неравенство $v_{\nu,-}\left(x_{0}\right)<a_{\nu}\left(x_{0}\right)$. В самом деле, если $0 \leqslant \nu<1$, то $v_{\nu,-}\left(x_{0}\right)=0<a_{\nu}\left(x_{0}\right)$, а если $\nu \geqslant 1$, то $\lim _{x_{0} \rightarrow 0} v_{\nu,-}\left(x_{0}\right)=$ $\nu-1<\nu=\lim _{x_{0} \rightarrow 0} a_{\nu}\left(x_{0}\right)$. Поэтому применение здесь части $\left.1^{0}\right)$ теоремы о дифференциальном неравенстве дает оценку $\sqrt{x^{2}+\nu^{2}}-1<a_{\nu}(x) \forall x>0, \nu \geqslant 0, \sqrt{x^{2}+\nu^{2}} \geqslant 1$.

III. Верхний барьер для $b_{\nu}(x)$. Таким барьером для $b_{\nu}(x)$ на полуотрезке $\left(0, x_{0}\right]$, где $0<x_{*}(\nu) \leqslant x_{0}, x_{*}(\nu)$ - теперь достаточно большое (зависящее от $\nu$ ), будет функция $w_{\nu,+}(x)=-\sqrt{x^{2}+\nu^{2}}$.

В самом деле, $w_{\nu,+}^{\prime}(x)=-x / \sqrt{x^{2}+\nu^{2}}, F_{\nu}\left(w_{\nu,+}(x), x\right) \equiv 0$, а потому $w_{\nu,+}^{\prime}(x)<$ $F_{\nu}\left(w_{\nu,+}(x), x\right) \forall x>0, \nu \geqslant 0$. Кроме того, в силу (20) при достаточно больших $x_{0}$

$$
w_{\nu,+}\left(x_{0}\right)=-x_{0}+O\left(x_{0}^{-1}\right)>b_{\nu}\left(x_{0}\right)=-x_{0}-\frac{1}{2}+O\left(x_{0}^{-1}\right) .
$$

Отсюда, применяя часть $2^{0}$ ) теоремы о дифференциальном неравенстве, получаем, что $b_{\nu}(x)<-\sqrt{x^{2}+\nu^{2}} \forall x>0, \nu \geqslant 0$.

IV. Построение нижнего барьера для $b_{\nu}(x)$. Этот барьер для $b_{\nu}(x)$ опять на полуотрезке $\left(0, x_{0}\right]$, где $x_{0}$ - достаточно большие, ищем в виде $w_{\nu,-}(x)=-\sqrt{x^{2}+\nu^{2}}-\beta$, $\beta>0$.

Согласно части $2^{0}$ ) теоремы о дифференциальном неравенстве $\beta$ нужно подобрать таким, чтобы неравенство $w_{\nu,-}^{\prime}(x)>F_{\nu}\left(w_{\nu,-}(x), x\right)$ выполнялось $\forall x>0$. Это неравенство эквивалентно неравенству

$$
x^{2}<2 \beta\left(x^{2}+\nu^{2}\right)+\beta^{2} \sqrt{x^{2}+\nu^{2}} .
$$

Легко видеть, что последнее неравенство вьполняется для любых $x>0$ и при любых $\nu \geqslant 0$ тогда и только тогда, когда $\beta \geqslant 1 / 2$. Кроме того, $\beta$ должно быть таким, чтобы 
при достаточно больших $x_{0}$ вьполнялось неравенство

$$
w_{\nu,-}\left(x_{0}\right)=-x_{0}-\beta-\frac{\nu^{2}}{2 x_{0}}+O\left(x_{0}^{-3}\right)<b_{\nu}\left(x_{0}\right)=-x_{0}-\frac{1}{2}-\frac{4 \nu^{2}-1}{8 x_{0}}+O\left(x_{0}^{-2}\right) .
$$

Такоенеравенство, как нетрудно видеть, вьполняется уже при минимальном из возможных значений $\beta: \beta=1 / 2$. Поэтому оценка $-\sqrt{x^{2}+\nu^{2}}-1 / 2<b_{\nu}(x)$ вьполняется $\forall x>0$, $\forall \nu \geqslant 0$.

Сформулируем установленное в виде леммы.

ЛЕмма 1. Для любых $x>0 u \nu \geqslant 0$ имеют место двусторонние оченки

$$
\begin{gathered}
\sqrt{x^{2}+\nu^{2}}-1<a_{\nu}(x)<\sqrt{x^{2}+\nu^{2}} \\
-\sqrt{x^{2}+\nu^{2}}-\frac{1}{2}<b_{\nu}(x)<-\sqrt{x^{2}+\nu^{2}} .
\end{gathered}
$$

Отметим, что в силу положительности функции $a_{\nu}(x)$ оценка снизу $(21)$ при $\sqrt{x^{2}+\nu^{2}} \leqslant 1$ не несет никакой информации. Кроме того, оценки снизу в (21) оказывается недостаточно для построения уточненного нижнего барьера для функции $a_{\nu}(x)$. Для этой цели установим следующую оценку.

Лемма 2. Для любых $x>0, \nu: 0 \leqslant \nu \leqslant \sqrt{2}$ имеет место оценка

$$
\sqrt{x^{2}+1}-1+\frac{\nu^{2}}{4}<a_{\nu}(x)
$$

ДокАЗАТЕЛЬСтво. Проверим, что функция $v_{\nu,-}(x)=\sqrt{x^{2}+1}-1+\nu^{2} / 4$ является нижним барьером для функции $a_{\nu}(x)$ при $0 \leqslant \nu \leqslant \sqrt{2}$ на полуотрезках $\left[x_{0}, \infty\right)$, где $x_{0}>0$ достаточно малые. Неравенство $v_{\nu,-}^{\prime}(x)<F_{\nu}\left(v_{\nu,-}(x), x\right)$ здесь эквивалентно неравенству

$$
0<\left(1-\frac{\nu^{2}}{2}\right)\left(x^{2}+1\right)-\left(2-\frac{3 \nu^{2}}{2}+\frac{\nu^{4}}{16}\right) \sqrt{x^{2}+1} \stackrel{\text { def }}{=} \eta(x, \nu)
$$

и оно выполняется $\forall x>0,0 \leqslant \nu \leqslant \sqrt{2}$. Это вытекает из представления

$$
\eta(x, \nu)=\left(1-\frac{\nu}{2}\right)^{2}\left(\sqrt{x^{2}+1}-1\right)^{2}+\frac{\nu^{2}}{2}\left(1-\frac{\nu^{2}}{8}\right) \sqrt{x^{2}+1}+\frac{\nu^{2}}{2} .
$$

Далее, при достаточно малых $x_{0}>0$ и $0<\nu \leqslant \sqrt{2}$

$$
v_{\nu,-}\left(x_{0}\right)=\frac{\nu^{2}}{4}+O\left(x_{0}^{2}\right)<a_{\nu}\left(x_{0}\right)=\nu+O\left(x_{0}^{2}\right) .
$$

При $\nu=0$, производя разложения до $O\left(x_{0}^{6}\right)$, получаем при достаточно малых $x_{0}>0$

$$
v_{0,-}\left(x_{0}\right)=\frac{1}{2} x_{0}^{2}-\frac{1}{8} x_{0}^{4}+O\left(x_{0}^{6}\right)<a_{0}\left(x_{0}\right)=\frac{1}{2} x_{0}^{2}-\frac{1}{16} x_{0}^{4}+O\left(x_{0}^{6}\right) .
$$

Таким образом, $v_{\nu,-}(x)$ является верхним барьером для $a_{\nu}(x)$ и оценка $(23)$ установлена.

Перейдем теперь к доказательству теоремы 1. 


\section{3. Уточненные двусторонние оценки для функций (1).}

I. Построение уточненного верхнего барьера для $a_{\nu}(x)$. Будем искать уточненные верхний барьер $v_{\nu,+}(x)$ и нижний барьер $v_{\nu,-}(x)$ для $a_{\nu}(x)$ в виде

$$
v_{\nu, \pm}(x)=\sqrt{x^{2}+\nu^{2}}-\frac{x^{2}}{2\left(x^{2}+\nu^{2}\right)}+\alpha_{ \pm} \cdot \frac{x^{2}}{\left(x^{2}+\nu^{2}\right)^{3 / 2}},
$$

где $\alpha_{ \pm}(x)$ - некоторые постоянные. Тогда

$$
\begin{aligned}
v_{\nu, \pm}^{\prime}(x)= & \frac{x}{\sqrt{x^{2}+\nu^{2}}}-\nu^{2} \frac{x}{\left(x^{2}+\nu^{2}\right)^{2}}+\alpha_{ \pm} \frac{\left(2 \nu^{2}-x^{2}\right) x}{\left(x^{2}+\nu^{2}\right)^{5 / 2}} \\
F_{\nu}\left(v_{\nu, \pm}(x), x\right)= & \frac{x}{\sqrt{x^{2}+\nu^{2}}}-\frac{\left[\left(2 \alpha_{ \pm}+0,25\right) x^{2}+2 \alpha_{ \pm} \nu^{2}\right] x}{\left(x^{2}+\nu^{2}\right)^{2}} \\
& +\alpha_{ \pm} \cdot \frac{x^{3}}{\left(x^{2}+\nu^{2}\right)^{5 / 2}}-\alpha_{ \pm}^{2} \frac{x^{3}}{\left(x^{2}+\nu^{2}\right)^{3}}
\end{aligned}
$$

Для верхнего барьера $v_{\nu,+}(x)$ должны выполняться условия

$$
\begin{aligned}
v_{\nu,+}^{\prime}(x) & >F_{\nu}\left(v_{\nu,+}(x), x\right) \quad \forall x>0, \quad \forall \nu \geqslant 0, \\
v_{\nu,+}\left(x_{0}\right) & >a_{\nu}\left(x_{0}\right) \quad \text { для достаточно малых } x_{0} .
\end{aligned}
$$

Неравенство (26) эквивалентно неравенству

$$
\left(\left(2 \alpha_{+}+\frac{1}{4}\right) x^{2}+\left(2 \alpha_{+}-1\right) \nu^{2}\right)+\alpha_{+} \frac{2\left(\nu^{2}-x^{2}\right)}{\sqrt{x^{2}+\nu^{2}}}+\alpha_{+}^{2} \frac{x^{2}}{x^{2}+\nu^{2}}>0 .
$$

Устремляя здесь $\nu \mathrm{k} \infty$, находим, что необходимым условием выполнимости неравенства $(28)$ при любых $\nu$ является условие $\left(2 \alpha_{+}-1\right) \geqslant 0$, т.е. условие $\alpha_{+} \geqslant 1 / 2$.

Положим значение $\alpha_{+}$минимальным из возможных, т.е. $\alpha_{+}=1 / 2$. С таким $\alpha_{+}$ неравенство (28) может быть приведено к виду

$$
\frac{1}{4} x^{2}+x^{2}\left(1-\frac{1}{2 \sqrt{x^{2}+\nu^{2}}}\right)^{2}+\frac{\nu^{2}}{\sqrt{x^{2}+\nu^{2}}}>0
$$

а потому заведомо вьполняется для любых $x>0, \nu \geqslant 0$. Следовательно, функция $v_{\nu,+}(x)$, определенная $(24)$ с $\alpha_{+}=1 / 2$, удовлетворяет условию (26).

Более того, такая $v_{\nu,+}(x)$ удовлетворяет и условию $(27)$. В самом деле, используя (19), имеем для $\nu>0$

$$
v_{\nu,+}\left(x_{0}\right)=\nu+\frac{1}{2}\left(\frac{1}{\nu}-\frac{1}{\nu^{2}}+\frac{1}{\nu^{3}}\right) x_{0}^{2}+O\left(x_{0}^{4}\right)>a_{\nu}\left(x_{0}\right)=\nu+\frac{1}{2(\nu+1)} x_{0}^{2}+O\left(x_{0}^{4}\right)
$$

при достаточно малых $x_{0}$, поскольку

$$
\left(\frac{1}{\nu}-\frac{1}{\nu^{2}}+\frac{1}{\nu^{3}}\right)=\frac{\left(1+\nu^{-3}\right)}{(\nu+1)}>\frac{1}{(\nu+1)} \forall \nu>0
$$

При $\nu=0$ также

$$
v_{0,+}\left(x_{0}\right)=x_{0}-\frac{1}{2}+\frac{1}{2 x_{0}}>a_{\nu}\left(x_{0}\right)=O\left(x_{0}^{2}\right)
$$

при достаточно малых $x_{0}>0$.

Таким образом, применяя часть $1^{0}$ ) теоремы о дифференциальном неравенстве, устанавливаем справедливость оценки сверху в (5). 
II. Построение уточненного нижнего барьера для $a_{\nu}(x)$. Значение параметра $\alpha_{-}$в выражении (24) для нижнего барьера $v_{\nu,-}(x)$ для $a_{\nu}(x)$ нужно подобрать так, чтобы выполнялись неравенства противоположного знака с (26), (27):

$$
\begin{gathered}
v_{\nu,-}^{\prime}(x)<F_{\nu}\left(v_{\nu,-}(x), x\right) \quad \forall x>x_{0}, \\
v_{\nu,-}\left(x_{0}\right)<a_{\nu}\left(x_{0}\right)
\end{gathered}
$$

где $x_{0}>0$ сейчас не конкретизируется и должно быть подобрано ниже.

Аналогично (28) неравенство (29) эквивалентно неравенству

$$
\left(\left(2 \alpha_{-}+\frac{1}{4}\right) x^{2}+\left(2 \alpha_{-}-1\right) \nu^{2}\right)+\alpha_{-} \frac{2\left(\nu^{2}-x^{2}\right)}{\sqrt{x^{2}+\nu^{2}}}+\alpha_{-}^{2} \frac{x^{2}}{x^{2}+\nu^{2}}<0 .
$$

Устремляя в этом неравенстве $x$ к $\infty$, находим, что необходимым условием его выполнимости является условие $\left(2 \alpha_{-}+1 / 4\right)<0$, т.е. условие $\alpha_{-}<-1 / 8$.

Положим в выражении (24) для $v_{\nu_{-}}(x) \alpha_{-}=1 / 2$. Тогда неравенство (31) приобретает вид

$$
\theta(x, \nu) \stackrel{\text { def }}{=}-2 \nu^{2}-\frac{\nu^{2}}{\sqrt{x^{2}+\nu^{2}}}-\frac{3}{4} x^{2}+\frac{x^{2}}{\sqrt{x^{2}+\nu^{2}}}+\frac{x^{2}}{4\left(x^{2}+\nu^{2}\right)}<0 .
$$

Покажем, что это неравенство вьполняется для любых $x>0, \nu \geqslant 1 / 2$. В самом деле, производя элементарные выкладки и оценки, имеем для любых $x>0$ (так как $\left.\sqrt{x^{2}+\nu^{2}}+\nu>2 \nu\right)$

$$
\begin{aligned}
\theta(x, \nu) & =-2 \nu^{2}-\nu+\frac{\nu x^{2}}{\sqrt{x^{2}+\nu^{2}}\left(\sqrt{x^{2}+\nu^{2}}+\nu\right)}-\frac{3}{4} x^{2}+\frac{x^{2}}{\sqrt{x^{2}+\nu^{2}}}+\frac{x^{2}}{4\left(x^{2}+\nu^{2}\right)} \\
& <-2 \nu^{2}-\nu-x^{2}\left(\frac{3}{4}-\frac{3}{2} \frac{1}{\sqrt{x^{2}+\nu^{2}}}-\frac{1}{4\left(x^{2}+\nu^{2}\right)}\right) \\
& =-2 \nu^{2}-\nu-x^{2}\left[\frac{3}{4}\left(1-\frac{1}{\sqrt{x^{2}+\nu^{2}}}\right)^{2}-\frac{1}{x^{2}+\nu^{2}}\right] \\
& \leqslant-2 \nu^{2}-\nu+\frac{x^{2}}{x^{2}+\nu^{2}}<-2 \nu^{2}-\nu+1 \leqslant 0
\end{aligned}
$$

при $\nu \geqslant 1 / 2$.

Более того, неравенство (32) вьполняется и для любых $x>0, \nu \geqslant 0$, удовлетворяющих условию $\sqrt{x^{2}+\nu^{2}}>(\sqrt{7}+2) / 3$. Действительно, это неравенство заведомо будет выполнено, если будет выполнено неравенство

$$
\frac{x^{2}}{4}\left(-3+\frac{4}{\sqrt{x^{2}+\nu^{2}}}+\left(\frac{1}{\sqrt{x^{2}+\nu^{2}}}\right)^{2}\right)<0
$$

и поскольку многочлен $z^{2}+4 z-3$ отрицателен на интервале $(-\sqrt{7}-2, \sqrt{7}-2)$, неравенство $(33)$, а вместе с ним и неравенство $(32)$ будет выполняться при $1 / \sqrt{x^{2}+\nu^{2}}<(\sqrt{7}-2)$, т.е. при $\sqrt{x^{2}+\nu^{2}}>1 /(\sqrt{7}-2)=(\sqrt{7}+2) / 3$. 
Перейдем к исследованию выполнимости неравенства (30). В случае $\nu \geqslant 1 / 2$ неравенство (30) выполняется для $x_{0}>0$ достаточно малых. В самом деле, в этом случае при достаточно малых $x_{0}$

$$
v_{\nu,-}\left(x_{0}\right)=\nu+\frac{1}{2}\left(\frac{1}{\nu}-\frac{1}{\nu^{2}}-\frac{1}{\nu^{3}}\right) x_{0}^{2}+O\left(x_{0}^{4}\right)<a_{\nu}\left(x_{0}\right)=\nu+\frac{1}{2(\nu+1)} x_{0}^{2}+O\left(x_{0}\right)^{4},
$$

поскольку

$$
\frac{1}{\nu}-\frac{1}{\nu+1}=\frac{1}{\nu(\nu+1)}<\frac{1}{\nu^{2}}<\frac{1}{\nu^{2}}+\frac{1}{\nu^{3}}
$$

В случае $0 \leqslant \nu<1 / 2$ возьмем в качестве $x_{0}>0$ точку, лежащую на окружности $\sqrt{x_{0}^{2}+\nu^{2}}=(\sqrt{7}+2) / 3$. Для таких $x_{0}$

$$
\begin{aligned}
v_{\nu,-}\left(x_{0}\right) & =\sqrt{x_{0}^{2}+\nu^{2}}-\frac{1}{2}+\frac{\nu^{2}}{2\left(x_{0}^{2}+\nu^{2}\right)}-\frac{1}{2 \sqrt{x_{0}^{2}+\nu^{2}}}+\frac{\nu^{2}}{2\left(x_{0}^{2}+\nu^{2}\right)^{3 / 2}} \\
& =\frac{2+\sqrt{7}}{3}-\frac{(\sqrt{7}-1)}{2}+\frac{1}{2}(\sqrt{7}-2)^{2}(\sqrt{7}-1) \nu^{2} \\
& =\frac{7-\sqrt{7}}{6}+\frac{15 \sqrt{7}-39}{2} \nu^{2} .
\end{aligned}
$$

С другой стороны, используя оценку (23), получаем

$$
a_{\nu}\left(x_{0}\right)>\sqrt{1-\nu^{2}+x_{0}^{2}+\nu^{2}}-1+\frac{1}{4} \nu^{2}=\sqrt{\frac{20+4 \sqrt{7}}{9}-\nu^{2}}-1+\frac{1}{4} \nu^{2} .
$$

Отсюда имеем

$$
\begin{aligned}
& v_{\nu,-}\left(x_{0}\right)-a_{\nu}\left(x_{0}\right) \\
& \quad<\left(\frac{7-\sqrt{7}}{6}+\frac{15 \sqrt{7}-39-0,5}{2} \nu^{2}-\left.\sqrt{\left.\frac{20+4 \sqrt{7}}{9}-\nu^{2}+1\right)}\right|_{\nu=0,5}<-0,0253<0,\right.
\end{aligned}
$$

поскольку $(15 \sqrt{7}-39,5) / 2>0,0931>0$ и функция, фигурирующая в круглых скобках, является монотонно возрастающей функцией $\nu$.

Таким образом функция $v_{\nu,-}(x)$ является нижним барьером для функции $a_{\nu}(x)$ на любом полуотрезке $\left[x_{0}, \infty\right)$, где $x_{0}>0$ - любое достаточно малое в случае $\nu \geqslant 1 / 2$ и $x_{0}>0$ лежит на окружности $\sqrt{x_{0}^{2}+\nu^{2}}=(\sqrt{7}+2) / 3$ в случае $0 \leqslant \nu<1 / 2$. Применяя часть $1^{0}$ ) теоремы о дифференциальном неравенстве, приходим к оценке снизу в (5) для функции $a_{\nu}(x)$ на множестве $(6)$.

III. Построение уточненного верхнего барьера для $b_{\nu}(x)$. Уточненные верхний барьер $w_{\nu,+}(x)$ и нижний барьер $w_{\nu,-}(x)$ для функции $b_{\nu}(x)$ на полуотрезках $\left(\sigma_{ \pm}, x_{0}\right]$, где $x_{0}>0$ - произвольное достаточно большое, $\sigma_{ \pm}=\sigma_{ \pm}(\nu)>0$, будем искать в виде

$$
w_{\nu, \pm}(x)=-\sqrt{x^{2}+\nu^{2}}-\frac{x^{2}}{2\left(x^{2}+\nu^{2}\right)}+\beta_{ \pm} \frac{x^{2}}{\left(x^{2}+\nu^{2}\right)^{3 / 2}}
$$


где $\beta_{ \pm}-$некоторые числа. Как и выше,

$$
\begin{aligned}
w_{\nu, \pm}^{\prime}(x)= & -\frac{x}{\sqrt{x^{2}+\nu^{2}}}-\nu^{2} \frac{x}{\left(x^{2}+\nu^{2}\right)^{2}}+\beta_{ \pm} \frac{\left(2 \nu^{2}-x^{2}\right) x}{\left(x^{2}+\nu^{2}\right)^{5 / 2}} \\
F_{\nu}\left(w_{\nu, \pm}(x), x\right)= & -\frac{x}{\sqrt{x^{2}+\nu^{2}}}+\frac{\left[\left(2 \beta_{ \pm}-0,25\right) x^{2}+2 \beta_{ \pm} \nu^{2}\right] x}{\left(x^{2}+\nu^{2}\right)^{2}} \\
& +\beta_{ \pm} \frac{x^{3}}{\left(x^{2}+\nu^{2}\right)^{5 / 2}}-\beta_{ \pm}^{2} \frac{x^{3}}{\left(x^{2}+\nu^{2}\right)^{3}}
\end{aligned}
$$

Для верхнего барьера $w_{\nu,+}(x)$ должны вьполняться условия

$$
\begin{gathered}
w_{\nu,+}^{\prime}(x)<F_{\nu}\left(w_{\nu,+}(x), x\right) \quad \forall x \in\left(\sigma_{+}, x_{0}\right), \\
w_{\nu,+}\left(x_{0}\right)>b_{\nu}\left(x_{0}\right) .
\end{gathered}
$$

Неравенство (36) эквивалентно неравенству

$$
\left(\left(2 \beta_{+}-\frac{1}{4}\right) x^{2}+\left(2 \beta_{+}+1\right) \nu^{2}\right)-\beta_{+} \frac{2\left(\nu^{2}-x^{2}\right)}{\sqrt{x^{2}+\nu^{2}}}-\beta_{+}^{2} \frac{x^{2}}{\left(x^{2}+\nu^{2}\right)}>0 .
$$

Устремляя в этом неравенстве $x$ к $\infty$, находим, что необходимым условием его выполнимости является условие $\left(2 \beta_{+}-1 / 4\right) \geqslant 0$, т.е. $\beta_{+} \geqslant 1 / 8$.

Положим $\beta_{+}$равньм минимально возможному значению, т.е. $\beta_{+}=1 / 8$. При таком $\beta_{+}$перепишем неравенство (38) в виде

$$
\frac{5}{4} \frac{\nu^{2}}{\sqrt{x^{2}+\nu^{2}}}\left(\sqrt{x^{2}+\nu^{2}}-\frac{1}{5}\right)+\frac{x^{2}}{4\left(x^{2}+\nu^{2}\right)}\left(\sqrt{x^{2}+\nu^{2}}-\frac{1}{64}\right)>0 .
$$

Последнее неравенство, а потому и неравенство $(36)$, выполняется при $\sqrt{x^{2}+\nu^{2}} \geqslant 1 / 5$, причем, как нетрудно видеть, это условие является необходимьм для его выполнимости при всех достаточно больших $\nu$.

Остается проверить, что при достаточно больших $x_{0}>0$ выполняется и условие (37). В самом деле, разлагая $w_{\nu,+}(x)$ по степеням $x^{-1}$ и используя разложение $(20)$, имеем для $x_{0}>0$ достаточно больших

$$
\begin{aligned}
w_{\nu,+}\left(x_{0}\right) & =-x_{0}-\frac{1}{2}-\frac{4 \nu^{2}-1}{8 x_{0}}+\frac{\nu^{2}}{2 x_{0}^{2}}+O\left(x_{0}^{-3}\right) \\
& >b_{\nu}\left(x_{0}\right)=-x_{0}-\frac{1}{2}-\frac{4 \nu^{2}-1}{8 x_{0}}+\frac{4 \nu^{2}-1}{8 x_{0}^{2}}+O\left(x_{0}^{-3}\right) .
\end{aligned}
$$

Таким образом, применяя часть $2^{0}$ ) теоремы о дифференциальном неравенстве, приходим к справедливости оценки сверху в (7) на множестве (8).

IV. Построение уточненного нижнего барьера для $b_{\nu}(x)$. Для нижнего барьера $w_{\nu,-}(x)$ для $b_{\nu}(x)$, имеющего выражение $(34)$, должны вьполняться условия

$$
\begin{gathered}
w_{\nu,-}^{\prime}(x)>F_{\nu}\left(w_{\nu,-}(x), x\right) \quad \forall x \in\left(\sigma_{-}, x_{0}\right) \\
w_{\nu,-}\left(x_{0}\right)<b_{\nu}\left(x_{0}\right)
\end{gathered}
$$


Неравенство (39) эквивалентно неравенству

$$
\left[\left(2 \beta_{-}-\frac{1}{4}\right) x^{2}+\left(2 \beta_{-}+1\right) \nu^{2}\right]-\beta_{-} \frac{2\left(\nu^{2}-x^{2}\right)}{\sqrt{x^{2}+\nu^{2}}}-\beta_{-}^{2} \frac{x^{2}}{x^{2}+\nu^{2}}<0 .
$$

Устремляя в нем $\nu$ к $\infty$, находим, что необходимым условием его выполнимости является условие $\left(2 \beta_{-}+1\right)<0$, т.е. условие $\beta_{-}<-1 / 2$. Далее, возьмем $\beta_{-}=-\beta$, где $\beta>1 / 2$. Запишем неравенство (41) в виде

$$
x^{2}\left(\left(2 \beta+\frac{1}{4}\right)+2 \beta \frac{1}{\sqrt{x^{2}+\nu^{2}}}+\beta^{2} \frac{1}{\left(x^{2}+\nu^{2}\right)}\right)+\frac{\nu^{2}(2 \beta-1)}{\sqrt{x^{2}+\nu^{2}}}\left(\sqrt{x^{2}+\nu^{2}}-\frac{2 \beta}{2 \beta-1}\right)>0 .
$$

Последнее неравенство, а вместе с ним и неравенство (39), будет выполняться $\forall x>0$, удовлетворяющих условию $\sqrt{x^{2}+\nu^{2}} \geqslant 2 \beta /(2 \beta-1)$.

Кроме того, тогда при достаточно больших $x_{0}$

$$
\begin{aligned}
w_{\nu,-}\left(x_{0}\right) & =-x_{0}-\frac{1}{2}-\frac{1}{2}\left(\nu^{2}+2 \beta\right) \frac{1}{x_{0}}+O\left(x_{0}^{-2}\right) \\
& <b_{\nu}\left(x_{0}\right)=-x_{0}-\frac{1}{2}-\frac{1}{2}\left(\nu^{2}-\frac{1}{4}\right) \frac{1}{x_{0}}+O\left(x_{0}^{-2}\right) .
\end{aligned}
$$

Следовательно, функщия $w_{\nu,-}(x)$ с $\beta_{-}=-\beta, \beta>1 / 2$, представляет собой нижний барьер для $b_{\nu}(x)$ при $\sqrt{x^{2}+\nu^{2}}>2 \beta /(2 \beta-1), x>0$, а потому справедлива оценка снизу в (7) на множестве (9). Теорема 1 полностью доказана.

4. Равномерные двусторонние оценки для функций $I_{\nu}(x)$ и $K_{\nu}(x)$.

Перейдем к доказательству теоремы 2 . Поскольку

$$
\begin{gathered}
\int \frac{\sqrt{x^{2}+\nu^{2}}}{x} d x=\sqrt{x^{2}+\nu^{2}}+\nu \ln \frac{x}{\nu+\sqrt{x^{2}+\nu^{2}}} \\
\int \frac{x}{\sqrt{x^{2}+\nu^{2}}} d x=\frac{1}{2} \ln \left(x^{2}+\nu^{2}\right), \quad \int \frac{x}{\left(x^{2}+\nu^{2}\right)^{3 / 2}} d x=-\frac{1}{\sqrt{x^{2}+\nu^{2}}}
\end{gathered}
$$

имеем

$$
v_{\nu, \pm}(x)=\frac{d}{d x} V_{\nu, \pm}(x), \quad w_{\nu, \pm}(x)=\frac{d}{d x} W_{\nu, \pm}(x),
$$

где $v_{\nu, \pm}(x)$ определены формулой $(24)$ с $\alpha_{ \pm}= \pm 1 / 2, w_{\nu, \pm}(x)$ определены формулой $(34)$ c $\beta_{+}=1 / 8, \beta_{-}=-\beta, \beta>1 / 2$,

$$
\begin{gathered}
V_{\nu, \pm}(x)=\sqrt{x^{2}+\nu^{2}}+\nu \ln \frac{x}{\nu+\sqrt{x^{2}+\nu^{2}}}-\frac{1}{4} \ln \left(x^{2}+\nu^{2}\right) \mp \alpha_{ \pm} \frac{1}{\sqrt{x^{2}+\nu^{2}}} \\
W_{\nu, \pm}(x)=-\left(\sqrt{x^{2}+\nu^{2}}+\nu \ln \frac{x}{\nu+\sqrt{x^{2}+\nu^{2}}}\right)-\frac{1}{4} \ln \left(x^{2}+\nu^{2}\right) \mp \beta_{ \pm} \frac{1}{\sqrt{x^{2}+\nu^{2}}} .
\end{gathered}
$$

Поэтому двусторонние оценки (5) и (7) (после деления на $x$ ) можем переписать в виде

$$
\begin{aligned}
\frac{d}{d x} V_{\nu,-}(x) & <\frac{d}{d x} \ln I_{\nu}(x)<\frac{d}{d x} V_{\nu,+}(x), \\
\frac{d}{d x} W_{\nu,-}(x) & <\frac{d}{d x} \ln K_{\nu}(x)<\frac{d}{d x} W_{\nu,+}(x),
\end{aligned}
$$


причем для тех значений $x$, при которых выполняются соответствующие им оценки $(5)$ и (7).

Проинтегрируем каждую из частей последних неравенств от $x$ до $A, A>x$, и воспользуемся формулой Ньютона-Лейбница. В результате получим двусторонние оценки

$$
\begin{gathered}
V_{\nu,-}(A)-V_{\nu,-}(x)<\ln I_{\nu}(A)-\ln I_{\nu}(x)<V_{\nu,+}(A)-V_{\nu,+}(x) \\
W_{\nu,-}(A)-W_{\nu,-}(x)<\ln K_{\nu}(A)-\ln K_{\nu}(x)<W_{\nu,+}(A)-W_{\nu,+}(x)
\end{gathered}
$$

откуда для любого $A>x$

$$
\begin{gathered}
e^{V_{\nu,+}(x)} I_{\nu}(A) e^{-V_{\nu,+}(A)}<I_{\nu}(x)<e^{V_{\nu,-}(x)} I_{\nu}(A) e^{-V_{\nu,-}(A)}, \\
e^{W_{\nu,+}(x)} K_{\nu}(A) e^{-W_{\nu,+}(A)}<K_{\nu}(x)<e^{W_{\nu,-}(x)} K_{\nu}(A) e^{-W_{\nu,-}(A)} .
\end{gathered}
$$

Но в силу (3) и (4)

$$
\begin{aligned}
\lim _{A \rightarrow \infty} I_{\nu}(A) e^{-V_{\nu, \pm}(A)} & =\lim _{A \rightarrow \infty} \frac{e^{A}}{\sqrt{2 \pi A}} \sqrt{A} e^{-A}\left(1+O\left(A^{-1}\right)\right)=\frac{1}{\sqrt{2 \pi}} \\
\lim _{A \rightarrow \infty} K_{\nu}(A) e^{-W_{\nu, \pm}(A)} & =\lim _{A \rightarrow \infty} \sqrt{\frac{\pi}{2 A}} e^{-A \sqrt{A}} e^{A}\left(1+O\left(A^{-1}\right)\right)=\sqrt{\frac{\pi}{2}}
\end{aligned}
$$

Поэтому, переходя в оценках (42) и (43) при фиксированных $x$ к пределу при $A \rightarrow \infty$, получаем соответственно двусторонние оценки (10) и (11).

\section{СПИСОК ЦИТИРОВАННОЙ ЛИТЕРАТУРЫ}

[1] Пальцев Б. В. О быстросходящихся итерационных методах с расщеплением граничных условий для многомерной системы типа Стокса. Периодические “течения" между параллельными стенками // Докл. РАН. 1992. Т. 325. № 5. С. 926-931.

[2] Пальцев Б. В. О быстросходящихся итерационных методах с неполным расщеплением граничных условий для многомерной сингулярно возмущенной системы типа Стокса // Матем. сб. 1994. Т. 185. № 4. С. 101-150.

[3] Пальцев Б. В. О быстросходящихся итерационных методах с полным расщеплением граничных условий для многомерной сингулярно возмущенной системы типа Стокса // Матем. сб. 1994. Т. 185. № 9. С. 109-138.

[4] Абрамов А. А., Ульянова В. И. Об одном методе решения уравнения типа бигармонического с сингулярно входящим малым параметром // ЖКВММФ. 1992. Т. 32. № 4. С. 567-575.

[5] Бейтмен Г., Эрдейи А. Высшие трансцендентные функции. Т. 2. М.: Наука, 1974.

[6] Справочник по специальным функциям. Под ред. М. Абрамовича и И. Стиган. М.: Наука, 1979.

[7] Ватсон Г. Теория бесселевых функций. Т. 1. М.: ИЛ, 1949.

[8] Olver F. W. J. The asymptotic expansion of Bessel functions of large order // Philos. Trans. Royal Soc. London. Ser. A. 1954. V. 247. P. 328-368.

[9] Олвер Ф. Асимптотика и специальные функции. М.: Наука, 1990.

[10] Хартман $\Phi$. Обькновенные дифференциальные уравнения. М.: Мир, 1970.

Вычислительный центр Р АН

Поступило

E-mail: palcev@ccas.ru

29.12.1997 